\title{
MANUEL GARCÍA Y SU ESCUELA VOCAL
}

\author{
Małgorzata Kubala \\ Universidad de Música Federico Chopin \\ https://doi.org/10.18778/8220-195-6.34
}

\section{Resumen}

El presente artículo está dedicado a Manuel García, tenor español, compositor y uno de los más sobresalientes profesores de canto de la primera mitad del siglo XIX. García basó su escuela vocal en el estilo italiano llamado belcanto, proveniente a su vez de los evirados, y desarrolló su técnica significativamente, gracias a sus cualidades vocales naturales y sus habilidades personales. Fue considerado un especialista en el estilo español e intérprete sin igual de Mozart y Rossini. Sus logros se vieron enriquecidos por sus tres hijos: Manuel Patricio García, María Malibrán y Pauline Viardot-García. El estudio de los textos legados por la familia García es una gran fuente de información para descubrir los principios de su escuela y lo que de ella persiste en la técnica vocal actual.

Palabras clave: Manuel García, escuela de canto, belcanto, interpretación, ópera.

\section{1.}

\section{La génesis de la escuela vocal de García}

En la historia de la música vocal, las primeras escuelas aparecieron en el siglo XVII, en los centros italianos de Venecia, Boloña y Nápoles. La escuela vocal más famosa fue desarrollada en Nápoles por el compositor y profesor Nicolo Porpora (1686-1768), que enseñó a grandes intérpretes como Carlo Broschi (llamado Farinelli) o Gaetano Majorano (conocido como Caffarello), ambos 
castrados. Los sucesores de Porpora continuaron su doctrina durante el siglo XIX, conocida en toda Europa como la escuela italiana de belcanto, atrayendo a cantantes de diferentes países. Entre ellos también se incluyen dos cantantes españoles: Manuel García e Isabel Colbrán.

Manuel del Pópulo Vicente Rodríguez García, nació en Sevilla, en el seno de una familia pobre. Comenzó su educación musical a los seis años en el coro de una parroquia. Posteriormente estudió armonía y composición con Antonio Ripa y Juan Almarcha. En 1792 debutó como tenor en el teatro de Cádiz, cantando por primera vez un programa repleto de música española, con sainetes y tonadillas. Pocos años después llegó a Madrid, donde actuó en el Teatro del Príncipe. También fue director artístico del Teatro Caños del Peral - para este teatro compuso sus primeras piezas escénicas.

Uno de los trabajos más conocidos de García en este periodo es la ópera-monólogo El poeta calculista, basado en formas tradicionales españolas: tonadillas y danzas. Estrenada en 1805 en Madrid, fue posteriormente presentada con éxito en París en 1808. En Madrid, García cantó el espectáculo acompañándose a sí mismo con una guitarra, mientras que en París pudo permitirse hacerlo con una orquesta. Uno de los más impresionantes fragmentos de la partitura es, sin duda, la canción Yo que soy contrabandista. La forma musical muestra claramente la procedencia del autor; en ella podemos reconocer fácilmente el ritmo ternario del polo y la oscilación entre escalas modales, tan características del estilo andaluz (Rodríguez, 2006: 160). García usó ornamentaciones específicas imitando los cantos tradicionales. Como se puede intuir, podía conjugar en sus interpretaciones la emisión del canto clásico con algunos efectos especiales del canto tradicional español, incluyendo el registro agudo de tenor (por encima del si natural), cantado con un sonido más abierto y brillante.

Esta obra está considerada como el manifiesto artístico de Manuel García, quien se identificaba a sí mismo con un contrabandista que vive fuera de la ley y de las normas sociales. La pieza retrata la libertad artística en sus cadencias y en la afirmación de la vida en sus exclamaciones de alegría (¡Ay, ay, ay!) como 
auténtica imagen de una persona libre. En una de las primeras críticas leemos: "García provenía de un país donde incluso la música folclórica ha de ser interpretada para embellecer y conmover al auditorio con un arte atrevido, ejecutado con gracia masculina..." (Radomski, 2004: 96). Lo que realmente impresionaba a la gente era la parte emocional de sus interpretaciones, así como los adornos, producto de su libre inspiración artística. Estos dos elementos eran los más importantes de su escuela vocal, basada en la creación, no en la reproducción pasiva.

\section{2. \\ El desarrollo de la técnica del bel canto. Cooperación con Rossini}

En 1811, García partió de España a Italia, llegando a Nápoles en enero de 1812. Siendo ya un cantante maduro y experimentado, comenzó sus estudios con el tenor Giovanni Anasani, considerado el último discípulo y continuador del estimado Nicolo Porpora. También acudió a las lecciones del famoso castrado Giovanni Battista Velluti (conocido como Giambattista). García trabajó principalmente en incrementar su proyección y el volumen de su voz. Uniendo sus habilidades naturales al conocimiento de los antiguos maestros italianos, García enriqueció y desarrolló su voz, que ya empezaba a ser intensa y rica en colores, y al mismo tiempo flexible en todos los registros. Debutó en el Teatro San Carlos con la ópera de Marco Portogallo Oro non compra amore (El oro no puede comprar el amor). Así mismo consiguió producir su propia obra, la ópera buffa en estilo italiano Il Califo di Bagdad (El Califa de Bagdad) cantando el papel principal (1813).

Cuando Gioacchino Rossini apareció en Nápoles en 1815, García ya era primer tenor. Fue invitado a cantar en el estreno de Elisabetta, regina d'Inghliterra (Isabel, reina de Inglaterra), escrita por Rossini para el comienzo de la temporada de invierno. Teniendo a su disposición la potente y resonante voz de García, Rossini construyó el papel de Norfolk para mostrar las posibilidades dramáticas del canto ornamentado, específicamente en el registro 
medio de la voz. El estreno resultó un éxito extraordinario que no se repitió, sin embargo, en otros lugares y con otros intérpretes. Como nota de interés, el papel principal de Elisabetta estuvo interpretado por la excelente soprano española Isabel Colbrán, que se convirtió en la esposa de Rossini en 1822.

Con el papel de Norfolk, García inició su larga cooperación con Rossini, gracias a la cual desarrolló su técnica vocal y su gusto musical. Las óperas de Rossini influenciaron el estilo de composición de García. Al mismo tiempo, sus habilidades vocales se convirtieron en una inspiración para el compositor italiano. En diciembre de 1815, Rossini firmó un contrato para un nuevo espectáculo en el Teatro Argentina en Roma. El libreto Il Barbiere di Siviglia (El Barbero de Sevilla), basado en la comedia de Beaumarchais, fue concluido con un mes de antelación al estreno, que finalmente tuvo lugar el 20 de febrero de 1816. El escaso tiempo del que dispuso Rossini para la preparación provocó las especulaciones en torno a la posible contribución de García en la pieza final. En el manuscrito de Rossini la parte de la guitarra de la primera canzone de Almaviva "Se il mio nome saper voi bramate" (Si mi nombre sabes qué anhelas), fue escrita por una mano distinta, lo que puede confirmar las sospechas de que García podría haber sido quien la escribiera. Igualmente, el diseño melódico de la línea vocal ha sido identificado como de procedencia andaluza (Mengibar, 2018: 60). Independientemente de la validez de estas connotaciones, el papel de Almaviva tiene algunos atributos del carácter y personalidad de García. De hecho se convirtió en una de sus mayores creaciones artísticas.

Tras el éxito de El Barbero de Sevilla, Rossini comenzó a trabajar en Otello, que se presentó en diciembre de 1816 en el Teatro del Fondo en Nápoles. A pesar de que García no pudo participar en el estreno, debido a sus obligaciones contractuales en París, el papel principal, de acuerdo con Stendal, el primer biógrafo de Rossini, fue escrito para él. García interpretó el papel de Otello por primera vez en París en 1821. Consecuentes representaciones en Londres (1823-1824) fueron recibidas con gran entusiasmo por parte de los críticos ingleses: "La interpretación de García de Otello es un gran regalo - con un vigor y un dinamismo que no tienen 
igual [...] no hay límites para el esplendor de su voz, que al mismo tiempo asciende triunfante sobre todo el tumulto de la escena, y se subyuga con una dulzura juguetona y corre sobre una infinita variedad de hermosos pasajes..." (Radomski, 2005: 173).

El Otello de Rossini es un trabajo innovador, donde el estilo florido encontró por primera vez un perfecto contrapeso con las expresiones dramáticas de las grandes escenas con recitativos. Inauguró el estilo dramático de la ópera italiana en el siglo XIX tardío. Además de Almaviva, Otello se convirtió en uno de los grandes papeles de García aplaudido no solo como un excelente cantante, sino también como gran actor. Instauró un nuevo estándar de interpretación, puesto que unía a la precisión vocal de los ornamentos y a la calidad de un enorme e impresionante sonido una dimensión dramática. Como resultado, era percibido por los críticos en París como un ejemplo de perfección absoluta que posteriormente se transformó en un rasgo característico de los cantantes de su escuela.

El repertorio vocal de García contenía prácticamente todos los papeles para tenor escritos por Rossini. Interpretó L'italiana in Algeri (Una italiana en Argel), Il turco in Italia (Un turco en Italia), Tancredi (Tancredo), Zelmira (Zelmira), Riccardo e Zoraide (Ricardo y Zoraida), Mathilde di Shabran (Matilde de Shabran) y La Cenerentola (La Cenicienta). También fue el primer europeo que participó en todas las óperas de Rossini y las óperas italianas de Mozart en los Estados Unidos y México (durante una tournée familiar realizada entre 1825 y 1826). Rossini influyó, además, en su estilo operístico: esto puede observarse en la última obra de García, El gitano por Amor, publicada en París alrededor de $1831 / 1832$. 


\section{El repertorio de Mozart. Escuela vocal en Londres}

Con excepción de las piezas de Rossini, García era famoso por su interpretación de papeles mozartianos. Representó el personaje principal de Don Giovanni por primera vez en 1820, en el Teatro Italiano de París. Posteriormente, cantó papeles típicos del repertorio para tenor: Tamino en Die Zauberflöte (La Flauta Mágica), Ferrando en Cosi fan tutte (Así son todas) pero también, y al mismo tiempo, el Conde en Le nozze di Figaro (Las bodas de Fígaro). Resulta interesante que García interpretase dos personajes de dos tipos distintos de categoría vocal: tenor lírico y barítono (El Conde Almaviva y Don Giovanni).

Como sabemos por la crítica, García alcanzó el esplendor interpretativo en el papel del libertino sevillano Don Juan. Tras el éxito en París, García lo representó en Londres (1825) y Nueva York (1826), donde dirigió el espectáculo bajo la influencia del libretista Lorenzo da Ponte. Para ajustar el rol del Don Juan a sus habilidades vocales transpuso algunas partes graves e introdujo ornamentos en las partes a solo [por ejemplo en la Serenata "Deh vieni alla finestra” (Ven a la ventana), en la segunda estrofa]. De acuerdo con la crítica, García fue ovacionado por sus largas respiraciones, su increíble precisión y energía, así como también por su perfecta dicción y dulzura en el sonido.

La capacidad de usar dos tipos distintos de voz con igual desarrollo tanto en el registro agudo como en el grave indica una habilidad inverosímil para los cantantes de la época. Curiosamente, esas mismas posibilidades eran características de sus hijas: María Malibrán y Pauline Viardot García. Ambas interpretaron papeles del repertorio de soprano y de mezzo-soprano, usando diferentes registros y colores de la voz. Marie-Pierre Escudier, una de las investigadoras de las actuaciones de García, escribió sobre él que era el único que sabía cómo desarrollar el registro de pecho al mismo tiempo que el de la cabeza, lo que le proporcionaba, a él y a sus alumnos, la posibilidad de utilizar voces con doble registro (Escudier, 1840: 36). 
En 1824 García fundó la Academia de Canto en la calle Dover en Londres. Lo anunció en la prensa como un programa hecho tanto para estudiantes como para cantantes profesionales. Organizó las clases en un tramo de seis días por semana. En el mismo año publicó Exercises and Method of Singing with the Accompaniment for the Piano Forte (Ejercicios y Método de canto con acompanamiento para Piano), que contiene ejercicios en pequeños grupos - cada uno de ellos para dominar un aspecto técnico diferente como notas largas, ornamentación, cromatismos, cadencias, entre otros. Dichos ejercicios eran la base de una práctica diaria. De este modo educaba la voz, independientemente del repertorio a interpretar (García, 1820: 8).

Exceptuando la técnica vocal, García prestaba mucha importancia al desarrollo de las habilidades musicales de sus pupilos. Exigía improvisaciones en fragmentos musicales concretos, interpretaciones profesionales de un texto dramático y perfecta pronunciación. Solía repetir a sus alumnos que ser un gran cantante significaba ante todo ser un buen músico. Otra de las cosas más importantes en las enseñanzas de García residía en la forma y el color de las vocales. Siendo español, basaba su canto en la articulación de vocales latinas que son claras y brillantes. Este detalle fue clave para encontrar un sonido que se proyectara y expandiera sin ruidos o aire. Poseyendo esta clase de sonido, tenía la capacidad de modificar los tonos de color de diferentes maneras, con un significado emocional diferente para cada tipo de música.

\section{4. \\ Los continuadores}

La escuela vocal creada por Manuel García continuó y se desarrolló con dos de sus hijos: Manuel Patricio Rodríguez García (1805-1906) y su hija menor Pauline Viardot-García (1821-1910). La hija mayor María Félicité García (1808-1836), conocida como María Malibrán, fue una cantante famosa pero murió en la cúspide de su carrera, sin dejar ningún trabajo teórico. Los dos hijos mayores aprendieron a cantar con su padre Manuel García. 
Pauline era aún muy joven cuando su padre falleció, con lo que no pudo recibir lecciones de canto como tales, ya que solo acompañaba al piano a los alumnos de su padre durante las clases. Sin embargo, Manuel García dejó para ella un cuaderno con ejercicios vocales que utilizó hasta su muerte.

La carrera vocal de Manuel Patricio fue corta e infructuosa por razones de salud. Participó en algunos espectáculos, organizados por su padre para la familia en Estados Unidos y México (durante su gira entre 1825-1827), cantando entre otros el papel de Fígaro en Il barbiere di Siviglia y Jago en el Otello de Rossini. También actuó como Leporello en Don Giovanni de Mozart. Tras su regreso a París, Manuel Patricio intentó continuar su carrera artística pero pronto cambió de idea y en 1830 se unió al ejército francés. Trabajó en hospitales militares donde estudiaba anatomía y fisiología de la laringe, dirigiendo experimentos con animales. En pocos años, se convirtió en un conocido profesor de canto y el conservatorio de París le otorgó esa titulación en 1835. Cinco años después, su tratado Traité complet de l'art du chant (Tratado Completo del Arte del Canto) fue publicado en París. En 1848, Manuel Patricio se mudó a Londres, donde enseñó en la Real Academia de Música entre 1850 y 1895. En 1854 inventó el laringoscopio - el primer artilugio que permitía observar el funcionamiento de las cuerdas vocales, lo que le granjeó fama internacional. En 1894, su último tratado, Hints on Singing (Indicios sobre el Canto), fue publicado en Londres.

El Traité complet de l'art du chant de Manuel Patricio es el primer tratado científico que contiene una metodología vocal integral. Trabajó en dicho tratado con el profesor y amigo de su padre - Giovanni Battista Velluti. Quería reproducir el método de su padre y le dio una base científica al añadir los resultados de sus investigaciones (García hijo, 1984: xvii). A pesar de las críticas del mundo académico, fue reconocido como el pedagogo más grande de su tiempo, incluso por encima de la fama de su padre.

Como adición importante a la escuela de Manuel García, hemos de mencionar igualmente dos libros publicados por su hija Pauline Viardot-García. Esta debutó en 1839 en el Covent Garden en el papel de Desdémona en el Otello de Rossini. En 1840 se casó 
con Louis Viardot - un amigo de la familia García y el director del Teatro Italiano en París. Muy pronto alcanzó una trayectoria internacional, cantando en San Petersburgo y en Londres; también visitó España, Polonia y Alemania. En su repertorio encontramos óperas de Gluck y de Mozart, también papeles escritos por compositores contemporáneos: Meyerbeer, Bellini, Donizetti, Gounod, Verdi y Wagner. Tras concluir su espectacular carrera, Pauline comenzó su actividad docente. Desde 1861 trabajó en la publicación de su École classique de Musique de Chant (Escuela Clásica de Música para Canto) - una serie de cuadernos para estudiantes que tenía previsto incluir más de 350 piezas de diferentes compositores destinadas a sus clases de canto. El trabajo aún no estaba concluido (Waddington, 2004: 9) pero el conservatorio de París aceptó dichos cuadernos como parte de su programa de enseñanza. Entre los años 1871-1875, Viardot enseñó en el conservatorio parisino, pero siguió dirigiendo su práctica privada en Baden-Baden y Londres. En 1880 publicó el libro en dos partes Une heure d'édude. Excercices pour voix de femme (Ejercicios para la voz de mujer), que contiene ejercicios para estudiantes y algunos consejos básicos que explican las reglas para la apropiada emisión vocal (Kubala, 2016: 64). Igualmente de valor son los problemas vocales incluidos en la correspondencia con su hermano.

La escuela de Manuel García continuó igualmente en sus alumnos, que actuaron en escenarios paralelamente a Malibrán y Viardot. Entre ellos se encuentran grandes cantantes como las sopranos Joséphine de Méric y Henriette Méric-Lalande, el tenor Adolphe Nouritt, considerado el seguidor de García en el escenario. Entre los alumnos de Manuel Patricio, las de mayor fama son la soprano sueca Jenny Lind y la actriz Marie Tempest, pero también otros dos excelentes profesores de canto: Camille Everardi y Mathilde Marchesi (quien fue la maestra de canto más conocida en las últimas décadas del siglo XIX). Incluidos entre los alumnos de Viardot podemos indicar a Marianne Brandt, la primera Kundry en el Parsifal de Wagner, la contralto Jeanne Gerville-Rèache y la afamada soprano Desirée Artôt.

La escuela de Manuel García no tiene solo valor histórico sino que su influencia se prolonga incluso hasta nuestros días. En el 
siglo XX encontramos la tercera generación, con cantantes como Beverly Sills y la madre de Joan Sutherland, ambas estudiantes de Marchesi. De acuerdo con la investigación dirigida por el departamento vocal en la universidad de música Federico Chopin, muchos de los elementos de la escuela de García están presentes en la metodología del canto actual. Su escuela es la perfecta combinación de experiencia práctica con investigación sistemática y la solución de problemas técnicos.

\section{Bibliografía}

Escudier, M. (1840). Études biographiques sur les chanteurs, contemporains, précédées d'une esquisse sur l'art du chant. Paris: Just Tessier.

García, M. (1820). Exercises pour la Voix. Paris: Lemoine de Ph. Petit et Ch. Boieldien.

García, M. hijo (1984 reprint). A Complete Treatise on the Art of Singing, part I and II (1847). New York: Da Capo Press.

Kubala, M. (2016). Dwanaście mazurków Chopina w transkrypcji Pauliny Viardot, Muzyczne aspekty fascynującej amitié amoureuse. Warszawa: Wydawnictwo Uniwersytetu Muzycznego Fryderyka Chopina.

Mengíbar, A.M. (2018). Los García. Una familia para el canto. Sevilla: Fundación Pública Andaluza Centro de Estudios Andaluces.

Radomski, J. (2004). Manuel García (1775-1832). Chronicle of the Life of bel canto Tenor at the Dawn of Romanticism. Oxford: Oxford University Press.

Rodríguez, F.J.G. (2006). "Manuel García: El Contrabandista y la imagen exótica de la música española”, en A.R. Ferrer y A.M. Mengíbar (eds.), Manuel García: de la tonadilla escénica a la ópera española (1775-1832). Cádiz: Servicio de Publicaciones de la Universidad de Cádiz, 159-174.

Waddington, P. (2004). The musical works of Pauline Viardot-Garcia (1821-1910): a chronological catalogue with an index of titles $\&$ a list of writers set and composers arranged. Upper Hutt, N.Z., Whirinaki Press. 